\title{
Identification and determination of chlorinated paraffins using multivariate evaluation of gas chromatographic data
}

\author{
Marie-Louise Nilsson ${ }^{\mathrm{a}}$, Staffan Bengtsson ${ }^{\mathrm{a}}$, Henrik Kylin ${ }^{\mathrm{a}, \mathrm{b}}$ * \\ ${ }^{a}$ Department of Aquatic Sciences and Assessment, Swedish University of Agricultural \\ Sciences, P.O. Box 7050, SE-75007 Uppsala, Sweden \\ ${ }^{\mathrm{b}}$ Department of Water and Environmental Studies, Linköping University, SE-58183 \\ Linköping, Sweden \\ *Corresponding author; henrik.kylin@liu.se
}

\begin{abstract}
Chlorinated paraffins (CPs) were found in the biodegradable fraction of source separated waste from Uppsala, Sweden. We identified and quantified the CPs by multivariate evaluation of gas chromatography-electron capture detection chromatograms. Using principal component analyses (PCA) we identified different types of CP-formulations and also obtain quantitative data. PCA yielded better identifications of individual CP-formulations than visual comparison of chromatograms. Partial least squares regression gave good calibration curves of the standards, but did not work for the waste samples. No source of CPs could be identified in the waste collection chain, and as the waste samples seemed to contain at least two different CPformulations the source was probably to be found in the waste material itself. The method was used to determine CPs in additional environmental samples, demonstrating that multivariate methods may be developed into a powerful tool for identification and quantification of complex mixture.
\end{abstract}

Key words: Principal component analysis, partial least squares regression, source separated waste, environmental quality criteria, food contamination

Capsule: Multivariate methods were used to identify and quantify chlorinated paraffins in complex chromatograms.

\section{Highlights}

- Technical chlorinated paraffins were identified and quantified from GC-ECD chromatograms using multivariate statistical methods.

- Chlorinated paraffins organic household waste could also be identified and quantified.

- Methods for noise reduction developed for near-infrared reflectance spectroscopy were useful also to enhance the evaluation of the chromatographic data of chlorinated paraffins.

- A possible source of the chlorinated paraffins was identified as citrus peel. 


\section{Introduction}

Chlorinated paraffins (CPs) have found many applications, but the main uses are as flame-retardant plasticizers in vinyl plastics and as high-temperature lubricants in metal-processing industry (Tomy et al., 1998; EuroChlor, 2010). Production of CPs commenced in 1930 and today the total world production is approximately $300 \mathrm{kt} / \mathrm{year}$ (EuroChlor, 2010). CPs are produced by chlorination of petroleum n-alkane fractions under UV irradiation. Depending on the number of carbons in the petroleum alkane fraction they are characterized as short $\left(\mathrm{C}_{10}-\mathrm{C}_{13}\right)$, medium $\left(\mathrm{C}_{14}-\mathrm{C}_{17}\right)$ or long $\left(\mathrm{C}_{20}-\mathrm{C}_{30}\right)$ chain, and further into subcategories depending on their mass percentage of chlorine into low (30-50\%), medium (50-60\%), or highly (60-70\%) chlorinated. Carbon chain length and chlorine content determine the properties of the individual CP product and vary depending on the specific use. The reaction has low positional selectivity and gives complex mixtures of alkanes with varying carbon chain length and varying and unspecific chlorination (Tomy et al., 1998), why CPs are among the most complex mixtures of environmental pollutants; a single sum formula can give rise to a very large number of isomers (Shojania, 1999).

Several countries have regulated the use of CPs as they are persistent in the environment and bioaccumulate (Stockholm Convention, 2010). The complex mixture of homologues and isomers is problematic as it renders the determination of CPs complicated (Tomy, 2010); any CP product will elute over a wide retention time window without baseline resolution (Figures S1-S2 in electronic supplement). In addition, other commonly found environmental contaminants will act as interferences as they overlap and obscure the chromatographic pattern of the CPs (and vice versa). Consequently, information on levels and fate of CPs in the environment is limited compared to other persistent organic pollutants, e.g., polychlorinated biphenyls (PCB), the production of which started at about the same time as CPs (Tomy et al., 1998). CPs have been reported from both terrestrial and aquatic ecosystems, and both high and low trophic levels and they occur in both biota and in abiotic compartments such as sediment and soil (Bayen et al., 2006; Santos et al., 2006).

Many different extraction and clean-up procedures for the determination of CPs have been described (Bayen et al., 2006; Santos et al., 2006). Because of the chromatographic behaviour of CPs, a particular complication is to remove compounds that obscure the identification of the CP product and interfere with the quantification. Short columns that allow the CPs to elute within a shorter retention time span have been used to achieve lower detection limits (Coelhan, 1999; Fridén et al., 2004), but will also exacerbate the problems with interfering compounds. Generally, most clean-up procedures consist of several liquid chromatographic steps (Bayen et al., 2006; Santos et al., 2006), including gel permeation and adsorption chromatography, sometimes with addition of, e.g., liquid chromatography with stationary phases with modified selectivity (Nilsson et al., 2001; Fridén et al., 2004), or ultraviolet (UV) irradiation (Friedman and Lombardo, 1975; Fridén et al., 2004). For the final qualitative and quantitative determination gas chromatography (GC) with electron capture detector (ECD) has often been used, but, due to the complexity of the CPs, GC coupled to high resolution mass spectrometry (HRMS) has become increasingly popular (Bayen et al., 2006; Santos et al., 2006; Tomy, 2010). However, a drawback with HRMS is the cost, why methods that allow good determinations with GC-ECD have been explored (Fridén et al., 2004). 
The lower cost of GC-ECD determinations would make it possible to obtain more environmental data, potentially increasing the understanding of the environmental fate of CPs by obtaining a higher statistical power. The drawback, however, is that in many cases an understanding of the distribution within homologue classes is desirable. A GCECD instrument will not yield this type of information, and multidimensional GCHRMS instruments may be necessary (Tomy, 2010).

In many parts of Europe composting and anaerobic digestion are increasingly important methods of handling the biodegradable fraction of household waste (Nilsson, 2000; Brändli, 2006). Not only is it a means of reducing the amount of waste that needs to be deposited or incinerated, but it is also a potential means of recirculating nutrients from urban areas to agriculture or horticulture, thus contributing to a sustainable development of society. However, to use the compost or digestate in cultivation a minimum quality must be ensured so that contaminants do not build up in the arable soil, potentially reaching the crops. Developing environmental quality criteria for compost was one of the goals of a multidisciplinary study initiated by the Faculty for Agriculture, Landscape Planning and Horticulture at the Swedish University of Agricultural Sciences. During the project, CPs were found in the biodegradable fraction of household waste from Uppsala, Sweden. As the concentrations were fairly high, it was important to trace the source of the contamination in order to avoid such contamination in the future. As CPs were not expected to be present in food waste in Sweden at the levels detected, the initial hypothesis was that the CPs originated from somewhere within the collection chain from the households to the waste handling plant. As this would be a technical source, we expected that the CP profile in the samples should be possible to attribute to a technical CP-formulation. We, therefore, undertook a project to determine the identity of the CP-formulation contaminating the household waste, to track the source of the contamination, and to map the circulation and fate of CPs in the waste handling system in Uppsala.

Here we report on experiments to use multivariate processing of GC-ECD data for the qualitative and quantitative determination of CPs. We investigated if it was possible to use principal component analysis (PCA) to explore differences between different types of CPs, and if it was possible to develop partial least square regression (PLSR) models to quantify CPs.

\section{Materials and methods}

\subsection{Solvents and chemicals}

Different commercial CP products of short and medium chain length, with chlorine content between 42 to 70\% (Table 1), were used as standards. Cyclohexane, acetone, and dichloromethane (LabScan, Stockholm, Sweden) and $n$-hexane (Merck Eurolabs, Spånga, Sweden) were of pesticide grade. Hydromatrix, (Varian, Harbor City, CA, USA), was bought from Scantec Lab (Partille, Sweden). Sodium sulphate (Merck Eurolabs) was heated to $600{ }^{\circ} \mathrm{C}$ over night, cooled and stored in a desiccator until used. Silica gel 60 (Merck Eurolabs) was washed with dichloromethane, dried at $130^{\circ} \mathrm{C}$ for at least 5 hours, cooled in a desiccator, deactivated with 1.5\% (w/w) deionised water, and stored under $n$-hexane. Concentrated sulphuric acid was from Merck Eurolabs. 
Standard solutions of CPs were prepared in cyclohexane (Table 1). Internal standards were 1,2,5,6,9,10-hexabromocyclododecane (HBCD), $\alpha, \alpha, \alpha^{\prime}, \alpha^{\prime}$-tetrachloro-o-xylene, 2,2',5,6'-Tetrachlorobiphenyl (PCB 53), and decachlorobiphenyl (PCB 209) (SigmaAldrich, Stockholm, Sweden). HBCD, being a halogenated alkane as are the CPs, was added as surrogate standard prior to extraction to estimate the total recovery. The other standards were added after clean-up and used 1) to calibrate the retention times and 2) to estimate the recovery of HBCD and thus also the CPs. A retention time standard consisting of technical grade DDT (app. 76\% p,p'-DDT [1,1,1-trichloro-2,2-bis-(4chlorophenyl)-ethane] and 20\% o,p'-DDT [1,1,1-trichloro-2-(2-chlorophenyl)-2-(4chlorophenyl)-ethane], Merck Eurolabs) in $n$-hexane was prepared to calibrate the liquid chromatographic fractionation (see below).

\subsection{Samples}

The biodegradable fraction of fresh source separated household waste from Uppsala, Sweden, was collected in the last week of February 1995 (Eklind et al., 1997). The subsamples were divided into three groups WA, WB and WC, with slight differences in the methods of extraction and clean-up. The waste samples were partially dried at ambient temperature before extraction. Homemade orange marmalade made in January 1995 was obtained from three separate households in the vicinity of Uppsala.

\subsection{Extraction and clean-up}

Detailed information on the clean-up procedures have been given by Nilsson (2000), and Nilsson et al. (2001). In summary, WA samples were extracted in a Soxhlet apparatus and WB samples in a Soxtec Avanti 2050 Auto System (Foss Tecator, Höganäs, Sweden), both with dichloromethane and with addition of the recovery standard HBCD prior to extraction. After extraction the solvent was changed to cyclohexane and the extract treated with concentrated sulphuric acid after which the organic phase was filtered (Acrodisc CR PTFE filter, $0.45 \mu \mathrm{m}, 25 \mathrm{~mm}$, Gelman Sciences, Ann Arbor, MI, USA). The filtered extract was further purified using size exclusion chromatography (SEC) with Bio-Beads S-X3 (200-400 mesh, Bio-Rad Laboratories, Hercules, CA, USA) as stationary phase. The SEC-fraction containing the CPs was cleaned-up further on a Nucleosil $5 \mathrm{NO}_{2}$ nitrophenylsilica column $(250 \mathrm{~mm}$, $4.6 \mathrm{~mm}$ ID, $5 \mu \mathrm{m}$ phase thickness, Jones Chromatography, Hengoed, Mid Glamorgan, Wales) coupled to a Nucleosil $5 \mathrm{NH}_{2}$ (10 mm, $4 \mathrm{~mm}$ ID, $5 \mu \mathrm{m}$ phase thickness) guard column, with n-hexane as eluent. The eluent flow was reversed between the peaks of $o, p^{\prime}$-DDT and $p, p^{\prime}$-DDT and a backflush fraction was collected containing the CPs without any PCBs (Kylin et al., 1996) and $\alpha, \alpha, \alpha^{\prime}, \alpha^{\prime}$ - tetrachloro-o-xylene was added.

WC samples were ground with hydromatrix and extracted in a Soxtec Avanti 2050 Auto System with acetone:dichloromethane (3:1) with addition of PCB 53. After size exclusion chromatography as above, final clean-up was fractionation on deactivated $(1.5 \%)$ silica gel where the least polar organochlorines were eluted with $n$-hexane and the CPs with $n$-hexane:toluene (65:35). PCB 209 was added prior to quantification.

The marmalade samples, two from each household, were macerated with an UltraTurrax (IKA, Staufen, Germany) until smooth. Subsamples of each marmalade sample were taken out and dried at $105^{\circ} \mathrm{C}$ until stable mass readings were obtained. The 
marmalade samples ( $2 \mathrm{~g}$ ) were mixed with concentrated sulphuric acid (6 g) in a test tube with Teflon-lined screw cap, left standing for $24 \mathrm{~h}$, and then extracted with $n$ hexane $(3 \times 6 \mathrm{ml})$ after addition of the recovery standard HBCD. After phase separation with centrifugation, the $n$-hexane extracts were pooled and the volume reduced under a slow stream of nitrogen, after which the extracts were processed further as sample groups WA and WB above.

\subsection{Gas chromatography with electron capture detection}

Quantification was done on a Agilent 6890 gas chromatograph (Agilent, Wilmington, DE, USA) with a micro-cell electron capture detector (ECD) equipped with a CP-Sil 5CB fused silica capillary column ( $7 \mathrm{~m}, 0.32 \mathrm{~mm}$ ID, $0.25 \mu \mathrm{m}$ phase thickness, Chrompack, Middelburg, the Netherlands). The temperature program was $90{ }^{\circ} \mathrm{C}(1$ $\mathrm{min}$ ), $30^{\circ} \mathrm{C} / \mathrm{min}$ to $180^{\circ} \mathrm{C}, 8{ }^{\circ} \mathrm{C} / \mathrm{min}$ to $270{ }^{\circ} \mathrm{C}$, isothermal for $15 \mathrm{~min}$. A Chromeleon chromatography data system (Gynkotek HPLC, Munich, Germany) was used for collection of chromatographic data. The chromatographic data was interpreted using multivariate methods as described below. The chromatographic data was also interpreted by traditional visual inspection of the chromatograms and quantified using total CP peak area and external standard calibration. In order to check the linearity between concentration and the ECD response for the different CPs used in this study, calibration curves based on integration of the total peak area (electronic supplement Figure S1 main panel) were prepared.

\subsection{Processing of chromatographic data and multivariate analysis}

Before the multivariate analysis, the chromatographic data were processed using the auxiliary programme ChromPro (BioTriMark, Uppsala, Sweden). The main steps were; noise reduction (Lee et al., 1991), baseline definition and subtraction, adjustment of retention times using a simplex procedure and finally data reduction (Andersson and Hämäläinen, 1994). The retention time span containing the CPs in the standards was selected from the chromatograms and the base-line defined manually (see electronic supplement, the inset in Figure S1 for an example). The noise reduction level was set to 5 , the most restrictive. The baseline was defined with input values $\sim 6$ and then if necessary modified so the total chromatographic pattern of CP was one peak. Since the CP-peak includes sub-areas (sections) which are baseline, smaller variations or noise, they were reduced into areas which contain systematic variation only (Andersson and Hämäläinen, 1994).

The processed chromatographic data were exported to the Unscrambler software (CAMO, Oslo, Norway) for multivariate analysis. In the multivariate data matrix, the data points in chromatograms constitute the variables (x) and the standards and samples the object (y). Latent variable projection methods, principal component analysis (PCA) and partial least square regression (PLSR) where used for the qualitative and quantitative interpretation of the chromatographic profiles. PLSR was selected as regression method as it provides robust quantitative results (Thomas and Haaland, 1990) by balancing the $\mathrm{x}$ - and $\mathrm{y}$-information and therefore reduces irrelevant $\mathrm{x}$-variation in the calibration model (Martens and Naes, 1989). Both PCA and PLSR models were based on full cross validation as the most efficient way of utilizing the objects (CAMO, 1996). In order to give all variables the same variance, the data were weighed using the weighting standardization option (1/SDev). Regression models were built using the 
standard solutions for each technical CP, in order to predict the concentrations of CPs in the waste samples. The root mean square error of prediction (RMSEP), a value expressing the uncertainty that can be expected when predicting concentrations using the regression models was calculated for each model. To investigate if it was possible to increase the performance of the projection methods, variables corresponding to interferences in the waste sample extracts were excluded from the data matrix manually before the multivariate analysis. The interferences were identified as all sharp peaks superimposed on the broad CP signal. The samples in the three sample groups contained different interferences why different variables had to be excluded from the individual group. The resulting data matrices for the three sample groups WA, WB and WC consisted of 336, 482, and 441 variables, respectively. We also investigated if the transformation method multiplicative scatter correction (MSC) made the data more suitable for multivariate analysis.

\subsection{Quality assurance}

The CP recovery was tested with fortified samples and varied between 70 and $90 \%$ depending on extraction and clean-up methods. The limit of quantification was set to the lowest standard concentration of each CP standard, which was also used as the limit of detection (Table 1). In addition, the final concentration of the samples extracts was in no case below the limit of quantification. To properly estimate the detection limit of each CP product in the samples is more complicated as it requires both qualitative and quantitative certainty and was not pursued further than to ensure that all processed data were $>15$ times above the estimated noise. The highest concentrations reported here may have been outside of the linearity of the method and should only be taken as indicative of very high concentrations. The waste samples were checked for the presence of the surrogate and internal standards before these were selected. Procedural blanks were run with each batch of samples processed.

\section{Results}

Chromatograms of the CP standards are shown in the electronic supplement, Figure S2. Principal component analysis (PCA) describes the variation in the processed chromatographic data of the CP standards well (Figure 1A). Most of the information or variation in the chromatographic data from the standard solutions was described by the first two principal components (PCs), which describe 66\% and 24\%, respectively. One PC describes variation related to the retention time/chromatographic pattern of the specific technical CP and the other variation related to concentration. In the PCA plot (Figure 1A) the CP standards appear in two main classes. A closer view of the variation between the individual CP standards within each main class is shown in Figures 1B-C. The linearity calculated from the integrated total peak areas for the different CPs was found to be well within the concentration range used in this study, with $\mathrm{R}^{2}$ of 0.972 0.997. The PLSR models for each of the CP standards describe a linear relationship between concentration and processed chromatographic data with $\mathrm{R}^{2}$ of $0.965-0.993$, but unfortunately the models did not work for the waste samples (see below).

The waste sample chromatograms contained varying interferences (electronic supplement, Figure S3). Group WA and WC samples were worst afflicted, while the WB samples, in comparison, were relatively free of interferences. In spite of the complication with the interferences, most of the variation was explained by the first two 
PCs once the variables corresponding to interferences in the waste sample extracts were excluded from the data matrix (Figure 2). The sum of the two first PCs for each of the three sample groups WA, WB and WC describe $96 \%, 89 \%$ and $85 \%$ respectively of the variation in the chromatographic data. Generally, Unscrambler suggested three PCs as optimal, but as most of the variation was explained by the two first PCs, the third PC was not considered further. Visualization of the information in the chromatographic data improved after elimination of the variables corresponding to the main interferences and after transformation of data by MSC as exemplified by the PCA plot for the sample group WC and CP standards A, D, G and H before (Figure 3) and after (Figure 2C II) elimination of variables and MSC transformation.

The PCA plots indicate that the $\mathrm{CP}$ in the waste samples is more similar to the $\mathrm{CP}$ standards in the main class/group containing CP A, D, G and H (Figure 2A I, B I, and C I). This was further emphasized in the PCA plots of the waste samples and standards CP A, D, G and H (Figure 2A II, B II and C II). In the PCA plots for WA the waste sample 12 overlaps with CP A (Figure 2A II). In the PCA plots for WB sample group, sample 1 and 12 overlap with CP A (Figure 2B II). In sample group WC, sample 7 overlaps with CP D and sample 9 with CP G (Figure 2C II). Thus, the contamination of the waste material must either have undergone partial transformation or, more likely, contains more than one CP-formulation. Visual inspection of the chromatograms (electronic supplement, Figure S3) also gives the impression of more than one CP-formulation in the waste samples, as the CP in samples 1 and 12 is similar to CP standard A, in sample 9 is similar to CP standards A or G, while the CP in sample 7 did not agree with any standard but was most similar to standard D.

The CP concentrations in waste samples (0.1-50 $\mu \mathrm{g} / \mathrm{g}$ dry mass) and marmalade (up to $600 \mu \mathrm{g} / \mathrm{g}$ dry mass) were quantified using the total chromatographic peak area and the PCA (Table 2). The PLSR models, although they worked well for the standard solutions, could not be used for quantification of CPs in the samples.

\section{Discussion}

Because of the large numbers of homologues and isomers, gas chromatograms of CPs are very complex with little easily discernible information. We, therefore, opted to use multivariate methods as these are powerful tools to evaluate, e.g., near-infrared reflectance spectra, which also contain little information that can be evaluated visually (Bengtsson et al., 2007; Martens and Naes, 1989). Multivariate evaluation of GC-ECD chromatograms can clearly be used for the identification and quantification of technical CPs. Identifying CP-formulations in environmental samples by PCA can be done with higher certainty than visually comparing individual chromatograms, as the differences between different CPs are emphasized in the PCA plot compared to in the chromatograms. It is also easier to obtain a general overview over several samples in a PCA plot, where each score corresponds to one chromatogram. The importance of reliable identification of which CP mixture is present in a sample before quantification was shown in an intercalibration study by Tomy et al. (1999), in which it was concluded that quantification of CPs using a different commercial CP products as standards, provided incorrect concentrations, despite the fact that a $\mathrm{CP}$ with a gas chromatographic profile similar to the one quantified was used. This in itself is a problem as there are many different CP formulations from different manufacturers (Fiedler, 2010). We have 
not found any reference to how many different CP products are in existence, but each formulation in all likelihood will have its own congener distribution, which is the case for, e.g., PCB products (e.g., Schulz et al., 1989). The problems CPs will be more complex than for PCBs due to the much larger number of CP-congeners. For this study we used eight different formulations, none of which corresponded fully to the CPformulations in the waste samples.

Processing of chromatograms with overlapping chromatographic peaks by eliminating analytical signals by multivariate methods reduces the noise level without a significant loss in statistical information (Faigle et al., 1991). Excluding variables corresponding to interferences in the waste sample extracts enhanced both the identification and quantification. Some of the interferences in the waste samples were dominant peaks in the chromatograms, and therefore contributed with significant "noise" or "irrelevant" information to the PC in the PCA. It is noteworthy that in the presence of moderate amounts of interferences, accurate identification of a technical CP-formulation is facilitated with PCA even when direct visual identification is not possible. However, for the WA, in which contaminant levels were higher than in the other sample groups, it seems that too much information was excluded as crucial information about the CPs was obscured (Figure 2A II).

Multiplicative signal correction (MSC) was developed to correct for light scattering variations in reflectance spectroscopy (Martens and Naes, 1989), but has also been used to remove interferences (CAMO, 1996). The information in the CP chromatograms became clearer after MSC, which shows that MSC is suitable for processing chromatographic data. However, the full potential of MSC in chromatography still remains to explore.

The quantifications of CPs in the waste samples using the PCA plot agree reasonably well with quantifications using the total peak area (Table 2) as long as the number of interfering peaks is limited. On the other hand, the concentration in the waste predicted with PLSR (data not presented) deviated very much from quantifications with PCA and total peak area. The reason for this deviation is probably that the PLSR models did not cover all the variation in chromatographic data, which is the most common of several phenomena that can affect the quality of a partial least square calibration (Martens and Naes, 1989). A possible further development would be to introduce additional clean-up methods not available to us, e.g., UV irradiation, to remove additional interferences and investigate if the PLSR models would work better.

The household waste samples seem to be contaminated with at least two types of CPs, one high chlorinated short chain and one or two medium chlorinated medium chain CPs. In the environmental monitoring of CP, most attention has been given the highly chlorinated short chain CPs because of their toxicity (Tomy et al., 1997; Tomy et al., 1999; Tomy et al., 2000). However, medium chlorinated medium chain CPs have the highest biomagnification potential (Fisk et al.,1999), which has lead to an increased interest in monitoring these (Tomy and Stern, 1999).

The fact that there are at least two different types of CPs in the waste means that a single source within the waste collection chain can be ruled out. Instead it is more likely 
that the source should be sought in the waste material itself. In such a case, waste of animal origin would be more likely than vegetable waste as meat and fish are found at higher trophic levels where more CP should have accumulated. However, another possible source was discovered some time after the waste study was completed. High levels (up to $600 \mu \mathrm{g} / \mathrm{g}$ dry mass) of a CP similar to standard A were found in marmalade home made in Uppsala 1995 and 1996 (Table 2). The waste samples contained substantial amounts of citrus peel, why it is conceivable that at least some of the CP found in the waste sample came with the citrus peels. Why there should be so much CP in citrus peel is unclear, but indicates some unregistered or illegal use of CP, e.g., as solvent or carrier for fungistatic compounds used for postharvest protection of the citrus produce. This finding demonstrates that the method has potential for more general use to detect and determine CPs in various environmental matrices.

\section{Conclusions}

Chlorinated paraffins are among the most complex environmental pollutants known. Therefore, multivariate methods should be especially useful to evaluate the chromatograms. Although there are still issues with the removal of interferences, the multivariate evaluation system allows rapid determination of these compounds using GC-ECD with an accuracy similar to or better than other quantification methods using GC-ECD. Depending on the questions investigated this is a cheap alternative to obtain data on CPs in the environment compared to methods using HRMS, but due to the complexity of CPs there will be a continuing need of HRMS-methods as well. As the multivariate evaluation requires a number of technical mixtures for calibration the system will obviously work best to quantify technical mixtures. The possibility to evaluate the composition of mixtures of CP formulation by multivariate methods, e.g., soft independent Modelling of Class Analogies (SIMCA), should also be explored (Wold \& Sjöström 1977). In theory, it should be possible with SIMCA to estimate the mixture percentage of two CP-formulations in a sample. If samples with environmentally aged CP profiles are analysed, the evaluation system should be able to identify the technical CP most suitable to use as standard for quantification. This in itself should enhance the quality of the quantitative data, and enable more precise estimation of the environmental fate of CPs. Further, with the continuing development of the computer capacity tied to analytical instrument, we see no reason why it should not be possible to eventually evaluate data from multidimensional GC- HRMS with multivariate statistical methods; this should greatly enhance the possibility to quantify different homologue groups and congeners of CPs in environmental samples. It should be noted that in this study we used several different programme packages, some originally not designed for the evaluation of chromatograms. For full utilization of multivariate statistics for this type of evaluation it would be beneficial if integrated programme packages were developed. The principles behind the system should also be applicable to the identification and quantification of other complex mixtures, with applications in environmental and forensic as well as other sciences.

\section{Acknowledgments}

Roger Andersson gave valuable advice on simplex focusing and chemometrics. An unknown reviewer gave valuable input to the manuscript. The project was funded by the Environmental Fund of the Swedish Civil Engineers Association. 


\section{References}

Andersson, R., Hämäläinen, M.D., 1994. Simplex focusing of retention times and latent variable projections of chromatographic profiles. Chemometrics and Intelligent Laboratory Systems 22, 49-61.

Bayen, S., Obbard, J.P., Thomas, G.O., 2006. Chlorinated paraffins: A review of analysis and environmental occurrence. Environment International 32, 915-929.

Brändli, R.C., 2006. Organic Pollutants in Compost and Digestate in Switzerland. Thesis, École Polytechnique Fédérale de Lausanne, Switzerland.

Bengtsson, S., Berglöf, T., Kylin, H., 2007. Near infrared reflectance spectroscopy (NIR) as a tool to predict pesticide sorption in soil. Bulletin of Environmental Contamination and Toxicology 78, 295-298.

CAMO, 1996. The unscrambler 6 user's guide. Camo AS, Trondheim, 372 pp.

Coelhan, M., 1999. Determination of short-chain polychlorinated paraffins in fish samples by short-column GC/ECNI-MS. Analytical Chemistry 71, 4498-4505.

EuroChlor, 2010. http://www.eurochlor.org/aboutparaffins, accessed 20 Aug 2010.

Eklind, Y., Beck-Friis, B., Bengtsson, S., Ejlertsson, J., Kirchmann, H., Mathisen, B., Nordkvist, E., Sonesson, U., Svensson, B. H., Torstensson, L., 1997. Chemical characterization of source-separated organic household wastes. Swedish Journal of Agricultural Research 27, 167-178.

Faigle, J.F., Poppi, R.J., Scarminio, I.S., Bruns, R.E., 1991. Multi-component principal component regression and partial least-squares analyses of overlapped chromatographic peaks. Journal of Chromatography A 539, 123-132.

Fiedler, H., 2010. Short-chain chlorinated paraffins: Production use and international regulations. In: J de Boer (ed.), Chlorinated Paraffins. Handbook of Environmental Chemistry 10:1-40. Springer, Berlin.

Fisk, A.T., Tomy, G.T., Muir, D.C.G., 1999. Toxicity of $\mathrm{C}_{10^{-}}, \mathrm{C}_{11^{-}}, \mathrm{C}_{12^{-}}$, and $\mathrm{C}_{14^{-}}$ polychlorinated alkanes to Japanese medaka (Oryzias latipes) embryos.

Environmental Toxicology and Chemistry 18, 2894-2902.

Fridén, U., Jansson, B., Parlar, H., 2004. Photolytic clean-up of biological samples for gas chromatographic analysis of chlorinated paraffins. Chemosphere 54, 1079-1083.

Friedman, D., Lombardo, P., 1975. Photochemical technique for the elimination of chlorinated aromatic interferences in the gas-liquid chromatographic analysis for chlorinated paraffins. Journal of the Association of Official Analytical Chemists 58, 703-706.

Kylin, H., Nordstrand, E., Sjödin, A., Jensen, S., 1996. Determination of chlorinated pesticides and PCB in pine needles - improved method for the monitoring of airborne organochlorine pollutants. Fresenius' Journal of Analytical Chemistry 356, 62-69.

Lee, T.A., Headley, L.M., Hardy, J.K., 1991. Noise reduction of gas chromatography/mass spectrometry data using principal component analysis. Analytical Chemistry 63, 357-360.

Martens, H., Naes, T., 1989. Multivariate Calibration. Wiley, New York.

Nilsson, M.-L., 2000. Occurence and fate of organic contaminants in wastes. Thesis, Swedish University of Agricultural Sciences.

Nilsson, M.-L., Waldebäck, M., Liljegren, G., Kylin, H., Markides, K., 2001. Pressurised Liquid Extraction (PLE) of Chlorinated Paraffins from the Biodegradable Fraction of Source Separated Household Waste. Fresenius' Journal of Analytical Chemistry 370, 913-918. 
Santos, F.J., Parera, J., Galceran, M.T., 2006. Analysis of polychlorinated n-alkanes in environmental samples. Analytical and Bioanalytical Chemistry 386, 837-857.

Schulz, D.E., Petrick, G., Duinker, J.C., 1989. Complete characterization of polychlorinated biphenyl congeners in commercial Aroclor and Clophen mixtures by multidimensional gas chromatography-electron capture detection. Environmental Science \& Technology 23, 852-859.

Shojania, S., 1999. The enumeration of isomeric structures for polychlorinated nalkanes. Chemosphere 38, 2125-2141

Stockholm Convention, 2010. www.pops.int, accessed 14 Sept. 2010.

Thomas, E.V., Haaland, D.M., 1990. Comparison of multivariate calibration methods for quantitative spectral analysis. Analytical Chemistry 62, 1091-1099.

Tomy, G.T., 2010. Analysis of chlorinated paraffins in environmental matrices: The ultimate challenge for the analytical chemist. In: J de Boer (ed.), Chlorinated Paraffins. Handbook of Environmental Chemistry 10:83-106. Springer, Berlin.

Tomy, G.T., Stern, G.A., Muir, D.C.G., Fisk, A.T., Cymbalisty, C.D., Westmore, J.B., 1997. Quantifying $\mathrm{C}_{10}-\mathrm{C}_{13}$ polychloroalkanes in environmental samples by highresolution gas chromatography/electron capture negative ion high-resolution mass spectrometry. Analytical Chemistry 69, 2762-2771.

Tomy, G.T., Fisk, A.T., Muir, D.C.G., 1998. Environmental chemistry and toxicology of polychlorinated n-alkanes. Review of Environmental Contamination and Toxicology 158, 53-128.

Tomy, G.T., Westmore, J.B., Stern, G.A., Muir, D.C.G., Fisk, A.T., 1999. Interlaboratory study on quantitative methods of analysis of $\mathrm{C}_{10}-\mathrm{C}_{13}$ polychloro-nalkanes. Analytical Chemistry 71, 446-451.

Tomy, G.T., Stern, G.A., 1999. Analysis of $\mathrm{C}_{14}-\mathrm{C}_{17}$ Polychloro-n-alkanes in environmental matrixes by accelerated solvent extraction-high-resolution gas chromatography/electron capture negative ion high-resolution mass spectrometry. Analytical Chemistry 71, 4860-4865.

Tomy, G.T., Muir, D.C.G., Stern, G.A., Westmore, J.B., 2000. Levels of $\mathrm{C}_{10}-\mathrm{C}_{13}$ Polychloro-n-alkanes in marine mammals from the Arctic and the St. Lawrence River estuary. Environmental Science and Technology 34, 1615-1619.

Wold, S., Sjöström, M., 1977. SIMCA: A method for analyzing chemical data in terms of similarity and analogy. In Kowalski, B.R., ed., Chemometrics Theory and Application, American Chemical Society Symposium Series 52, Wash., D.C., American Chemical Society, p. 243-282. 
Table 1. Standard solutions of chlorinated paraffins (CP) with different carbon chain length $(\mathrm{C})$ and chlorine content $(\% \mathrm{Cl})$. The individual CP-products were designated with letters A-H. The standard solutions were fortified with 1, 2, 5, 6, 9, 10hexabromocyclododecane $(0.185 \mathrm{ng} / \mathrm{mL})$ and $\alpha, \alpha, \alpha^{\prime} \alpha^{\prime}$-tetrachloro-o-xylene $(0.019$ $\mathrm{ng} / \mathrm{mL}$ ) as surrogate and internal standards and were also used to calibrate the retention times using the auxiliary programme ChromPro.

\begin{tabular}{llllllll}
\hline & & \multicolumn{7}{c}{ Chlorinated paraffin } \\
$\mathrm{A}^{\mathrm{a}}$ & $\mathrm{B}^{\mathrm{a}}$ & $\mathrm{C}^{\mathrm{b}}$ & $\mathrm{D}^{\mathrm{b}}$ & $\mathrm{E}^{\mathrm{c}}$ & $\mathrm{F}^{\mathrm{c}}$ & $\mathrm{G}^{\mathrm{c}}$ & $\mathrm{H}^{\mathrm{c}}$ \\
$\mathrm{C}_{14-17}$ & $\mathrm{C}_{10-13}$ & $\mathrm{C}_{10-13}$ & $\mathrm{C}_{10-13}$ & $\mathrm{C}_{10-13}$ & $\mathrm{C}_{14-17}$ & $\mathrm{C}_{14-17}$ & $\mathrm{C}_{14-17}$ \\
$52 \%$ & $56 \%$ & $60 \%$ & $\begin{array}{c}70 \% \\
\text { Concentration }(\mu \mathrm{g} / \mathrm{mL})\end{array}$ & $42 \%$ & $52 \%$ & $57 \%$ \\
\hline 26.38 & 25.07 & 19.05 & 6.61 & 25.6 & 32.0 & 25.6 & 25.6 \\
19.33 & 12.53 & 9.52 & 3.31 & 12.8 & 25.6 & 12.8 & 12.8 \\
9.67 & 6.27 & 4.76 & 1.65 & 6.4 & 12.8 & 6.4 & 6.4 \\
4.83 & 5.48 & 4.17 & 1.45 & 5.6 & 6.4 & 5.6 & 5.6 \\
4.23 & 3.92 & 2.98 & 1.03 & 4.0 & 5.6 & 4.0 & 4.0 \\
3.02 & 2.35 & 1.79 & 0.62 & 2.4 & 4.0 & 2.4 & 2.4 \\
1.81 & 0.78 & 0.60 & 0.21 & 0.8 & 2.4 & 0.8 & 0.8 \\
0.60 & 0.39 & 0.30 & 0.10 & 0.4 & 0.8 & 0.4 & 0.4 \\
\hline
\end{tabular}

${ }^{\mathrm{a}}$ product from ICI Chlor-Chemicals (Milbank, UK)

${ }^{\mathrm{b}}$ product from Hüls (Marl, Germany)

${ }^{\mathrm{c}}$ Dr. Ehrenstorfer reference material (Augsburg, Germany) 
Table 2. Concentration of chlorinated paraffins in samples from the biodegradable fraction of household waste and homemade marmalade from Uppsala. The concentrations were determined in two different ways; estimated from the PCA plot and calculated from total chromatographic peak area for the CP. Samples with severe influence of interferences are italicized.

\begin{tabular}{|c|c|c|}
\hline $\begin{array}{l}\text { Sample group } \\
\text { and subsamples }\end{array}$ & $\begin{array}{l}\text { Calculated from the } \\
\text { integrated total peak area } \\
\text { ( } \mu \mathrm{g} / \mathrm{g} \text { dry mass) }\end{array}$ & $\begin{array}{l}\text { Estimated with PCA } \\
(\mu \mathrm{g} / \mathrm{g} \text { dry mass) }\end{array}$ \\
\hline \multicolumn{3}{|c|}{ 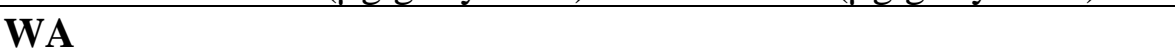 } \\
\hline 2 & 2.0 & $-^{\mathrm{a}}$ \\
\hline $5 b$ & 1.9 & $-^{\mathrm{a}}$ \\
\hline 11 & 1.2 & $-^{\mathrm{a}}$ \\
\hline 12aI10 & 38 & 47 \\
\hline 12aII10 & 48 & 42 \\
\hline 12b10 & 20 & 22 \\
\hline \multicolumn{3}{|l|}{ WB } \\
\hline $1 \mathrm{aL}$ & 6.9 & 3.0 \\
\hline $1 \mathrm{aH}$ & 6.8 & 2.4 \\
\hline $12 \mathrm{aH}$ & 47 & 45 \\
\hline $12 \mathrm{aL}$ & 38 & 27 \\
\hline $12 \mathrm{bH}$ & 20 & 26 \\
\hline $12 \mathrm{bL}$ & 25 & 20 \\
\hline \multicolumn{3}{|l|}{ WC } \\
\hline 3 & 2.1 & 0.72 \\
\hline 4 & 1.8 & $-^{\mathrm{a}}$ \\
\hline 6 & 1.1 & 1.2 \\
\hline 7 & 1.3 & 0.33 \\
\hline 8 & 0.50 & 0.10 \\
\hline 9 & 2.5 & 4.6 \\
\hline 10 & 0.8 & 0.32 \\
\hline 13 & 1.3 & 0.12 \\
\hline 14 & 1.7 & 0.09 \\
\hline \multicolumn{3}{|l|}{ Marmalade } \\
\hline $1 \mathrm{a}$ & 430 & 440 \\
\hline $1 b$ & 620 & 590 \\
\hline $2 \mathrm{a}$ & 160 & 140 \\
\hline $2 b$ & 170 & 140 \\
\hline 3a & 190 & 220 \\
\hline $3 b$ & 140 & 140 \\
\hline
\end{tabular}

${ }^{\mathrm{a}}$ PCA estimation failed 

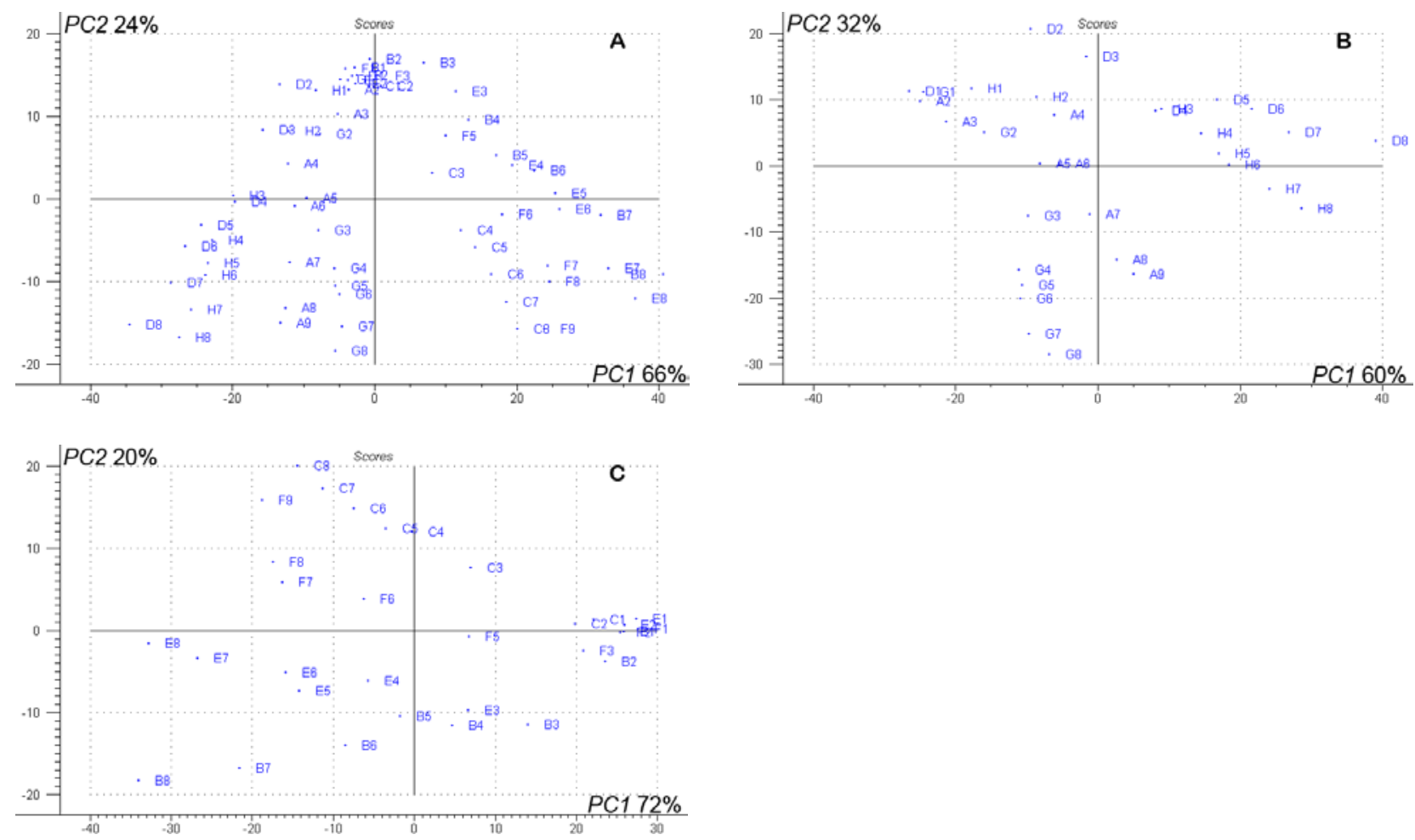

Figure 1. Principal component analysis (principal component 1 vs principal component 2) of the GC-ECD chromatograms of the CP standard solutions. Each type of technical CP gives calibration curves in different orientations in the PCA-plot. The percentage variation explained by each $\mathrm{PC}$ is given on the respective axis.
A. PCA of CPs A, B, C, D, E, F, G and H
B. Detailed PCA of CPs A, D, G and H
C. Detailed PCA of CPs B, C, E and F 

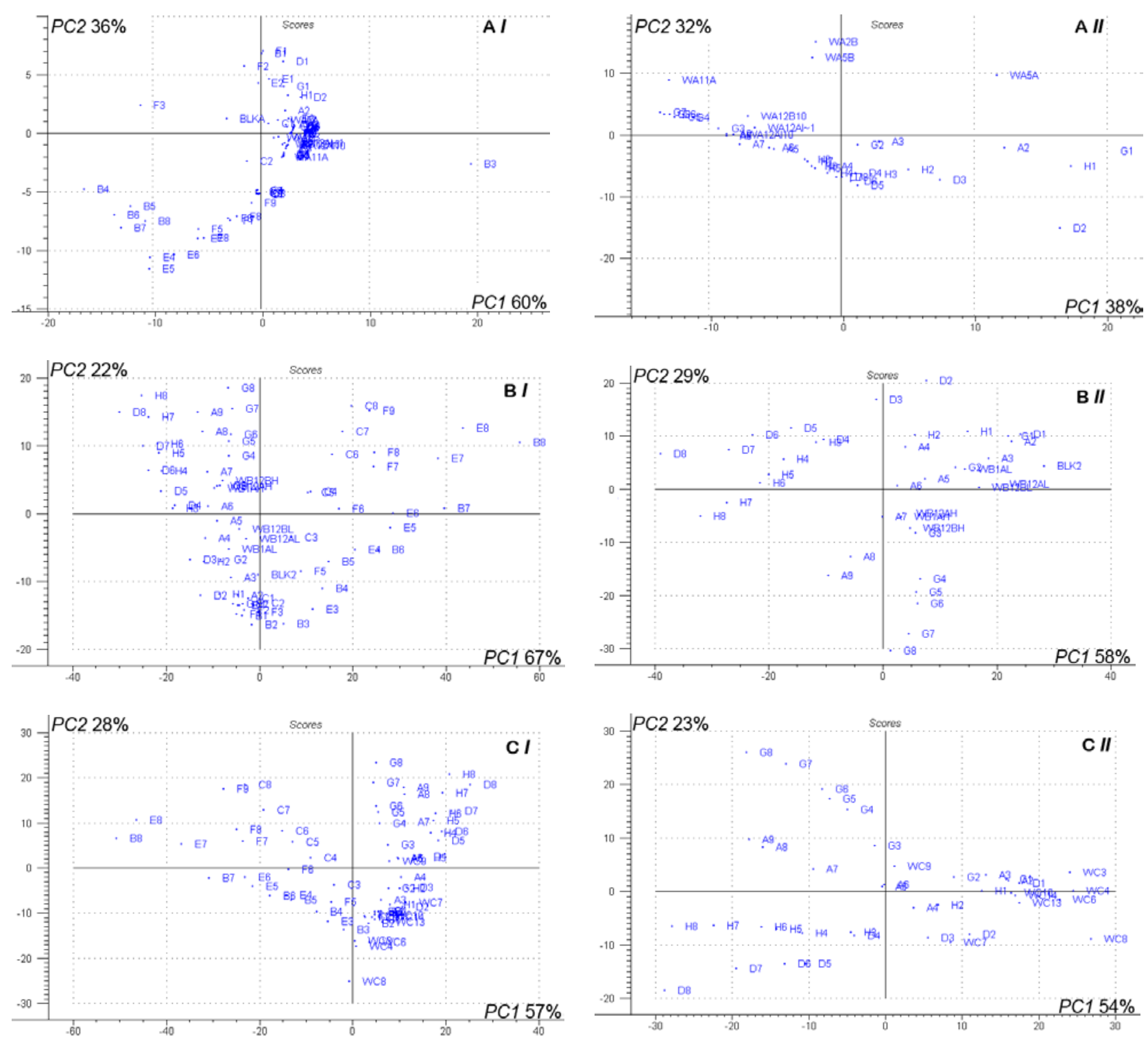

Figure 2. Principal component analysis (PC1 vs. PC2) of GC-ECD chromatograms of CP standard solutions and CPs in source separated household waste samples after data transformation by multiplicative scatter correction (MSC). Variables corresponding to interferences in the waste samples were excluded from the data. The percentage variation explained by each $\mathrm{PC}$ is given on the respective axis.

A. Sample group WA and CP A, B, C, D, E, F, G and H (I); A, D, G and H (II)

B. Sample group WB and CP A, B, C, D, E, F, G and H (I); A, D, G and H (II)

C. Sample group WC and CP A, B, C, D, E, F, G and H (I); A, D, G and H (II) 


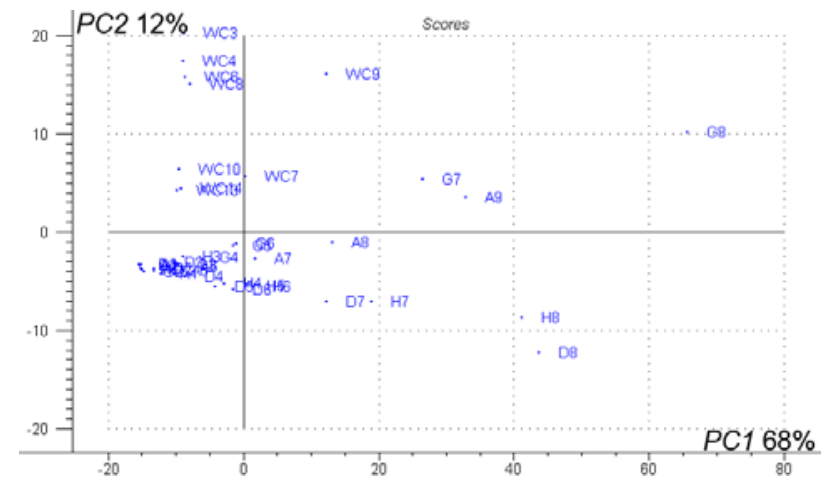

Figure 3. Principal component analysis (PCA) of chromatographic data from gas chromatography-electron capture detection analysis chlorinated paraffin (CP) standard solutions and source separated household waste samples WC. All variables were included in the data and the data was not transformed by MSC. The percentage variation explained by each PC is given on the respective axis. 
Electronic supplemental material

\title{
Identification and determination of chlorinated paraffins using multivariate evaluation of gas chromatographic data
}

\author{
Marie-Louise Nilsson ${ }^{\mathrm{a}}$, Staffan Bengtsson ${ }^{\mathrm{a}}$, Henrik Kylin ${ }^{\mathrm{b}}$ * \\ ${ }^{a}$ Department of Aquatic Sciences and Assessment, Swedish University of Agricultural \\ Sciences, P.O. Box 7050, SE-750 07 Uppsala, Sweden \\ ${ }^{\mathrm{b}}$ Department of Water and Environmental Studies, Linköping University, SE-581 83 \\ Linköping, Sweden
}

Figure S1. Examples of the integration methods of a short-chain high-chlorinated paraffin standard used in this study. Main panel: Total CP-peak area integration. Inset: Example of how the base line was drawn for the integration using multivariate evaluation of the chromatogram. Note that in the latter case only a small section of the total integrated area is shown.

Figure S2. Chromatograms of the chlorinated paraffin standards with added hexabromododecane and $\alpha, \alpha, \alpha^{\prime}, \alpha^{\prime}$ - tetrachloro-o-xylene. Products from Chlor-Chemicals (Milbank, UK): CP A, $\mathrm{C}_{14-17} 52 \% \mathrm{Cl}$; CP B, $\mathrm{C}_{10-13} 56 \%$ Cl. Products from Hüls (Marl, Germany): CP C, $\mathrm{C}_{10-13} 60 \% \mathrm{Cl}$; CP D, $\mathrm{C}_{10-13} 70 \% \mathrm{Cl}$. Reference materials from Dr. Ehrenstorfer (Augsburg, Germany): CP E, $\mathrm{C}_{10-13} 55.5 \% \mathrm{Cl}$; CP F, $\mathrm{C}_{14-17} 42 \% \mathrm{Cl}$; CP G, $\mathrm{C}_{14-17}$ $52 \% \mathrm{Cl} ; \mathrm{CP} \mathrm{H}, \mathrm{C}_{14-17} 57 \% \mathrm{Cl}$.

Figure S3. Chromatograms of selected waste samples. 
Figure S1. Examples of the integration methods of a short-chain high-chlorinated paraffin standard used in this study. Main panel: Total CPpeak area integration. Inset: Example of how the base line was drawn for the integration using multivariate evaluation of the chromatogram. Note that in the latter case only a small section of the total integrated area is shown.

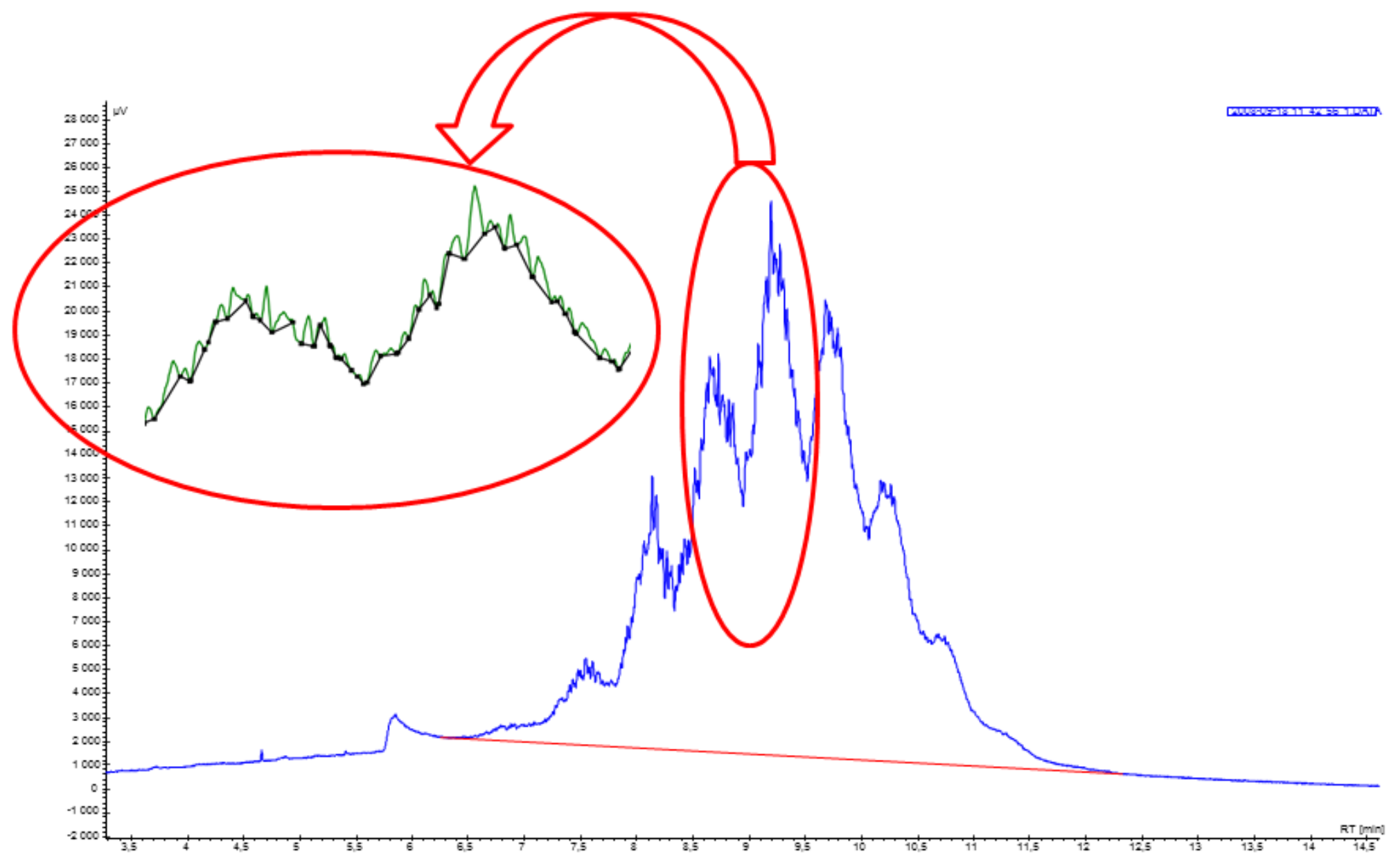


Figure S2. Chromatograms of the standard chlorinated paraffins with added hexabromododecane and $\alpha, \alpha, \alpha^{\prime}, \alpha^{\prime}$ - tetrachloro-o-xylene.
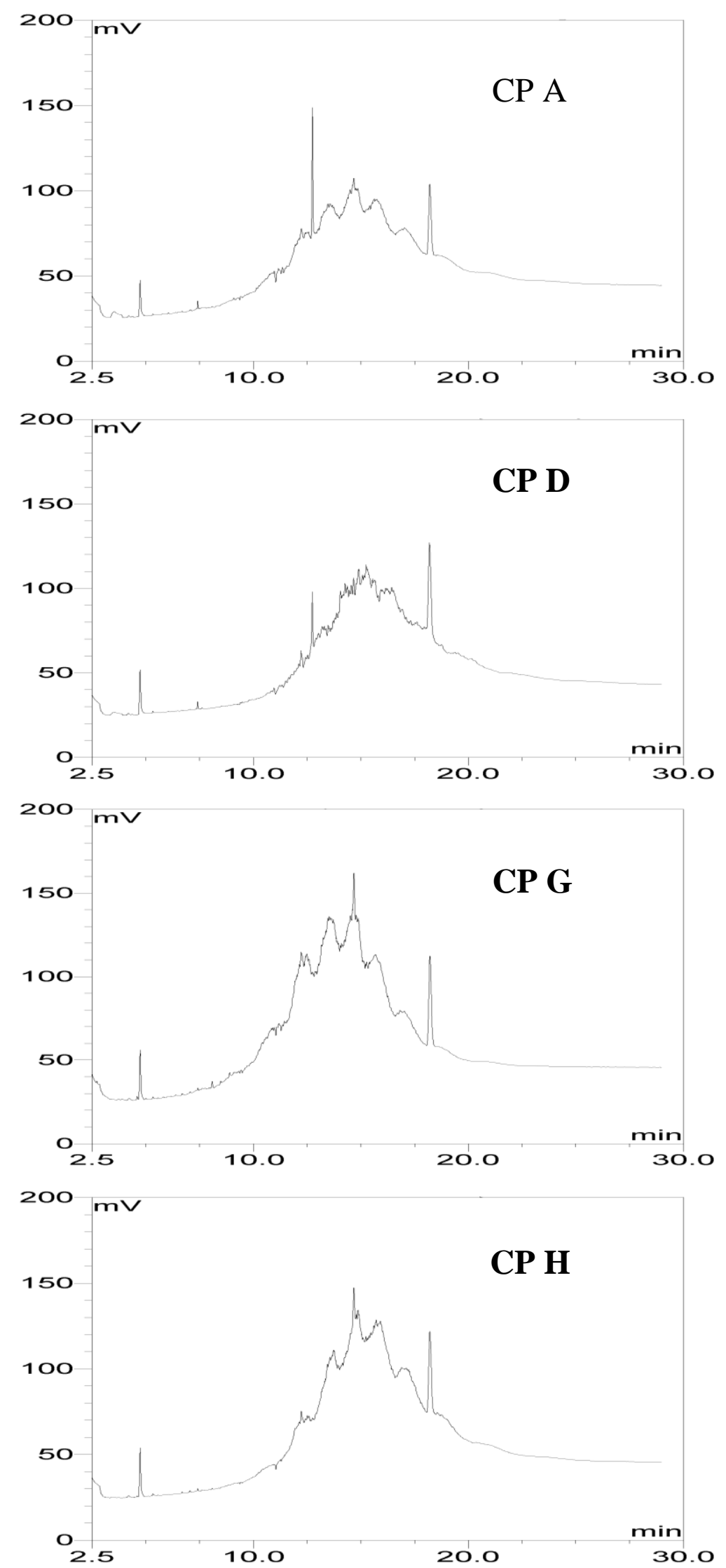

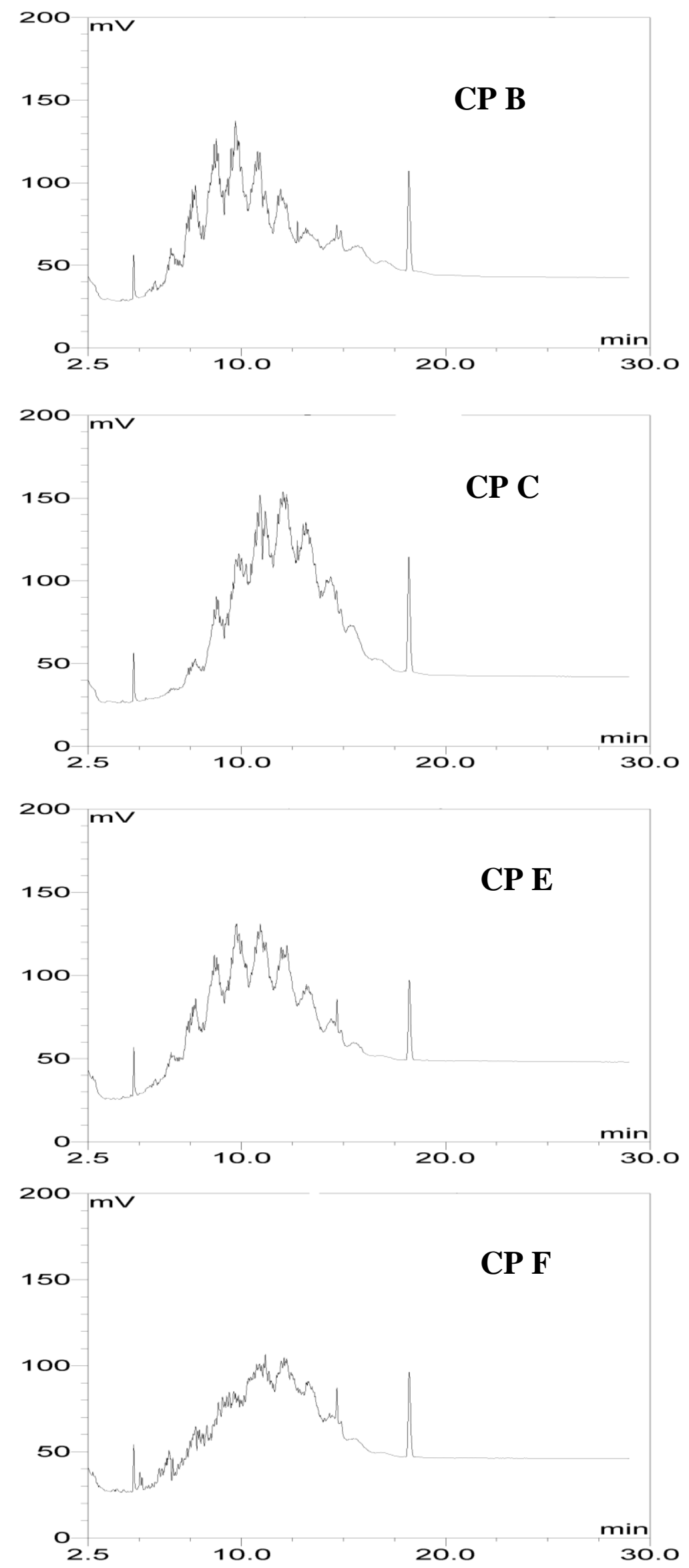
Figure S3. Chromatograms of selected waste samples.

WC
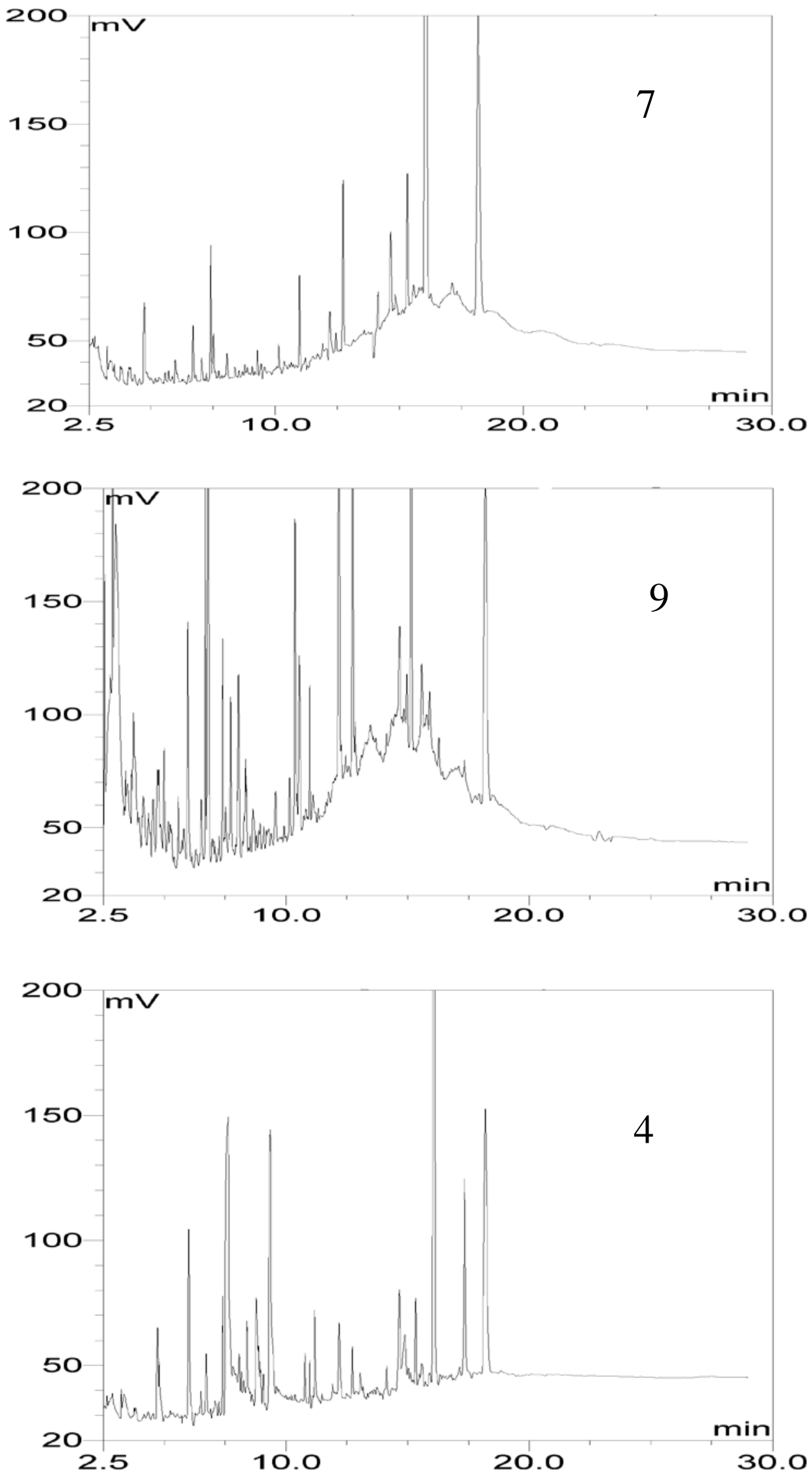


\section{WB}
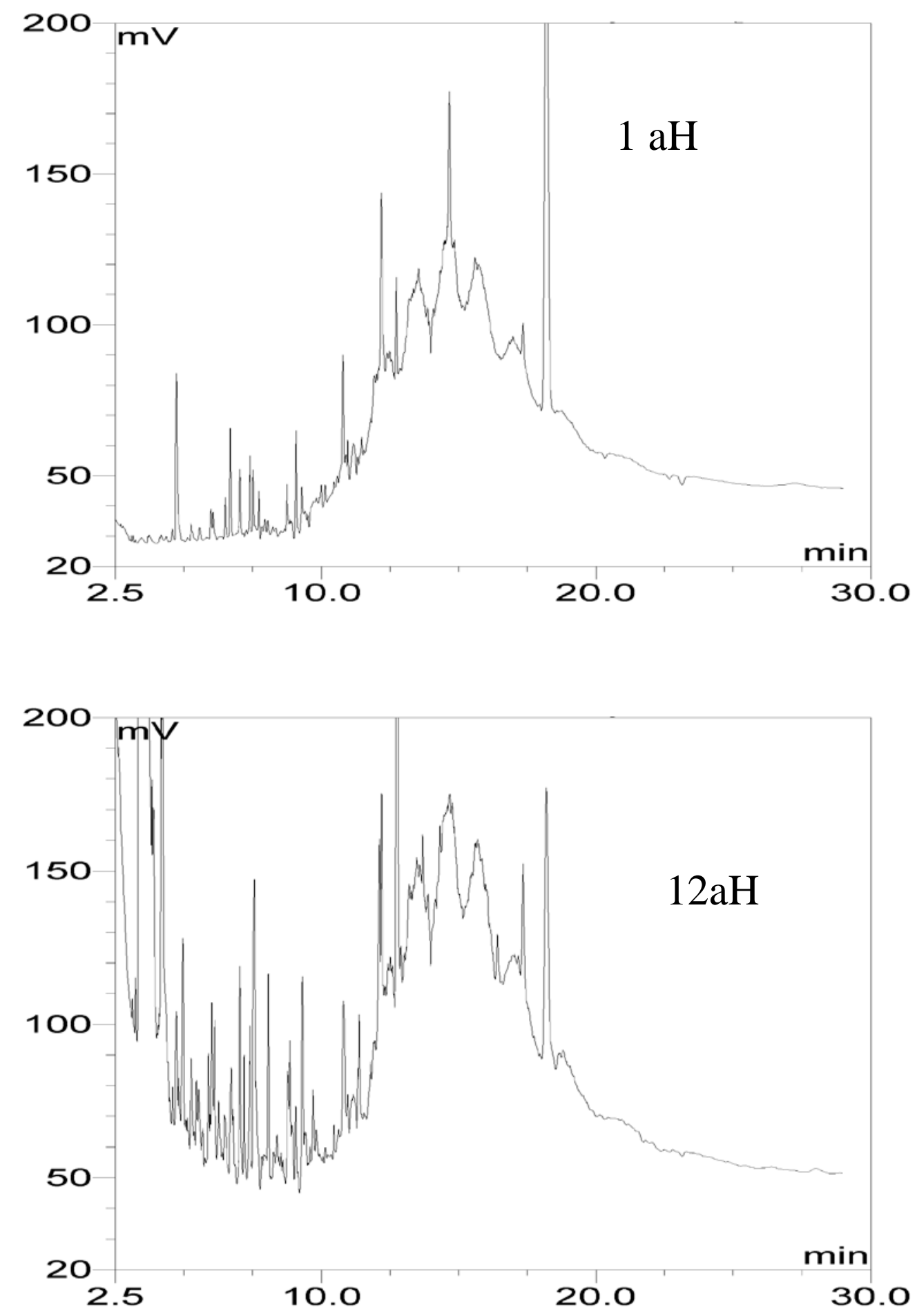
WA
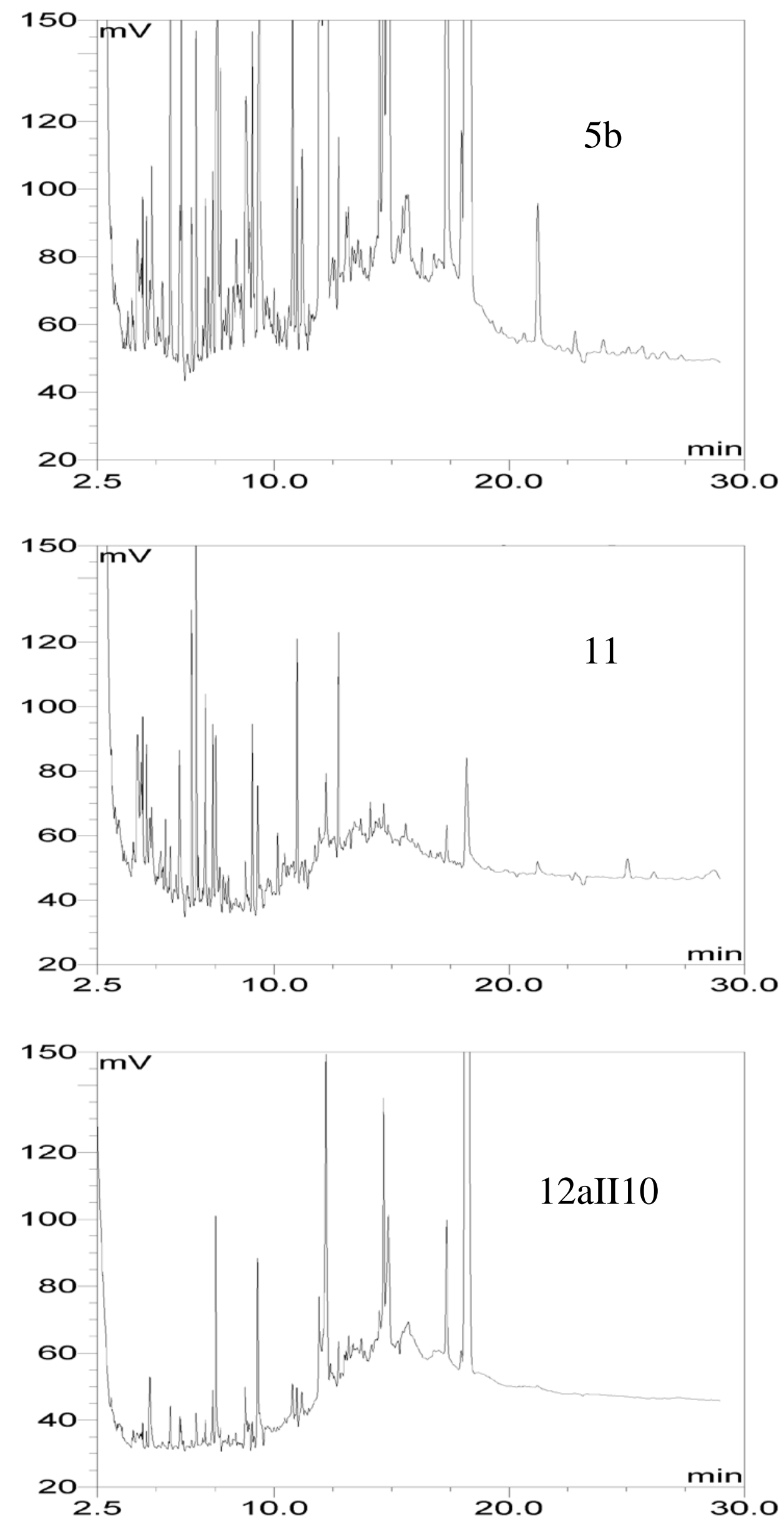